\title{
Fallpauschalen in Schweizer Spitälern - eine erste Bilanz
}

Der Ansatz der IDoC-Studie ist es, «harte» Daten - Indikatoren für Patientensicherheit und Daten aus klinischen Registern - mit den Wahrnehmungen von Ärzten, Pflegenden und Spitalmanagern zu kombinieren. Ein erstes Studienergebnis: Ärzte sehen keine Effizienzsteigerung seit Einführung von SwissDRG, aber eine nachlassende Patientenorientierung, verfrühte Entlassungen und auch andere Formen der Unter- und Überversorgung.

\section{Nikola Biller-Andorno}

Prof. Dr. med. Dr. phil., Direktorin Institut für Biomedizinische Ethik, Universität Zürich, Gesamtleitung IDoC-Studie

Lesen Sie zu diesem Thema auch das «Zu guter Letzt» von Rouven Porz.
Korrespondenz:

Prof. Dr. med. Dr. phil. Nikola Biller-Andorno Institut für Biomedizinische Ethik Universität Zürich Pestalozzistrasse 24 CH-8032 Zürich

biller-andorno[at]ethik.uzh.ch
Es ist zwei Jahre her, dass in der Schweiz im Zuge der Teilrevision des Krankenversicherungsgesetzes (KVG) Fallpauschalen (Diagnosis Related Groups) eingeführt wurden. Das erklärte Ziel der DRG-Einführung war ein Beitrag zur Kosteneindämmung durch wirtschaftliche Anreize, die bewirken, «dass unternehmerische Kriterien im Spitalbereich vermehrt zum Tragen kommen» [1]. Dabei sollten, so darf man annehmen, die Errungenschaften des KVG - ein qualitativ hochstehendes, umfassendes Leistungsangebot im Bereich der obligatorischen Krankenversicherung - nicht zur Disposition gestellt werden.

Die ökonomische Effizienz zu steigern, ohne Einbussen an Qualität und Fairness in der Versorgung hinnehmen zu müssen, ist eine enorme Herausforderung und zurzeit das grosse gesundheitspolitische Thema in vielen Ländern. Die DRG sind damit ein Ansatz neben anderen wie Accountable Care Organi- wenig mager. Drastische, unmittelbare Qualitätseinbussen waren von Anfang an unwahrscheinlich in einem System, im dem nicht nur die ethischen Standards von Ärzten und Pflegenden, sondern auch hohe Erwartungen von Patienten und Angehörigen einer solchen Entwicklung entgegenwirken. Die Gefahr besteht vielmehr in einer subtilen Verschiebung der Prioritäten in Spitälern vom Patientenwohl und der bedarfsgerechten Versorgung der gesamten Bevölkerung hin zu Erlössteigerung bzw. der Vermeidung von Verlusten, verbunden mit der Entwicklung neuer Formen von Über- und Unterversorgung.

Zugleich stellt sich die Frage, wie gut die empirische Datenlage ist, um Aussagen über Qualität und Fairness treffen zu können. Zwar werden begrüssenswerte Anstrengungen unternommen, um Versorgungsforschung und Qualitätsmessungen zu fördern, doch ist die aktuelle Datenbasis zu etwaigen Veränderungen unter DRG noch recht schmal.

\section{Die Gefahr besteht in einer subtilen Verschiebung der Prioritäten in Spitälern vom Patientenwohl und der bedarfsgerechten Versorgung hin zur Erlössteigerung.}

zations, bundled payments, den Choosing Wisely-Initiativen oder der integrierten Versorgung.

War die Einführung der SwissDRG nun ein Erfolg? Die kurze Antwort ist: Wir wissen es noch nicht. Bislang hört man wenig, dass durch die DRG die stationäre Versorgung oder - noch wichtiger die Gesundheitsversorgung insgesamt billiger geworden sei. Was man hört, ist, dass keine grösseren Katastrophen geschehen sind [2, 3]. Das ist zwar beruhigend, wäre als Ergebnis der Reform jedoch ein

\begin{abstract}
Ansatz und erste Ergebnisse der IDoC-Studie Der Ansatz der im Sinergia-Programm des Nationalfonds geförderten interdiziplinären Studie «Assessing the Impact of DRGs on Patient Care and Professional Practice» (IDoC) besteht darin, «harte» Daten - Indikatoren für Patientensicherheit und Daten aus klinischen Registern - mit den Wahrnehmungen professioneller Gruppen - von Ärzten, Pflegenden und Spitalmanagern - zu kombinieren. Ein Grund für dieses Vorgehen ist die Tatsache, dass eine eindeutige kau-
\end{abstract}




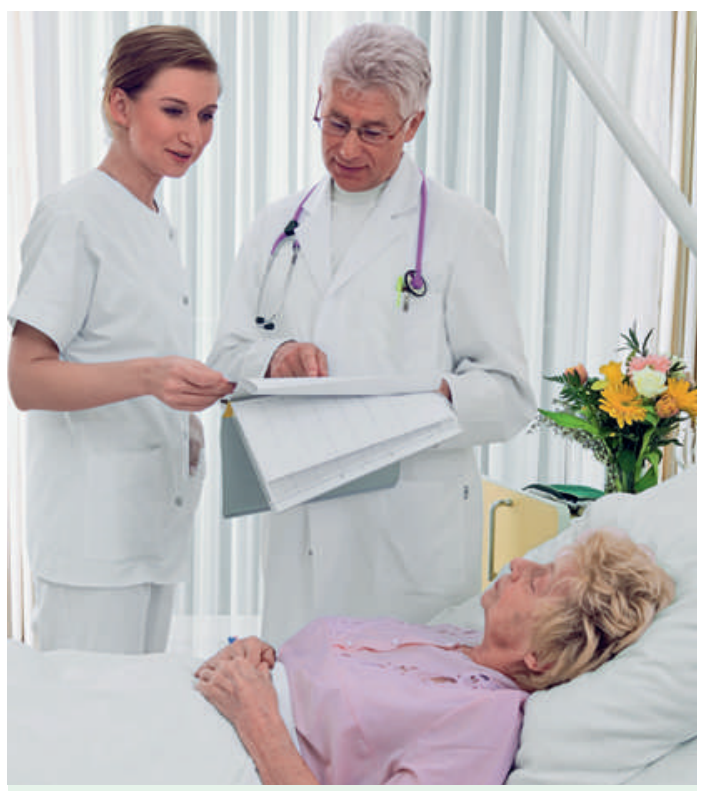

Ein Thema der IDoC-Studie: Wie nehmen die Vertreter der verschiedenen medizinischen Berufe den Spitalalltag seit Einführung der DRGs wahr?

sale Zuschreibung von Veränderungen in der Versorgungsqualität und der DRG-Einführung schwierig ist. Die Frage, ob Personen, die in ihrem Berufsalltag mit DRGs zu tun haben, bestimmte Veränderungen mit diesem Erstattungssystem in Verbindung bringen, kann zumindest ein wichtiges Indiz für einen solchen Zusammenhang liefern.

Hinzu kommt, dass sich Veränderungen nicht unbedingt in den klassischerweise erhobenen Qualitätsparametern abbilden, da Ärzte und Pflege gegen die DRG-Logik «anarbeiten». Was man in einem solchen Fall jedoch sehen würde, wäre eine Belastung durch moralische Konflikte und letztlich auch eine sinkende Arbeitszufriedenheit oder einen Mangel an Arbeitskräften. Längerfristig ist auch denkbar, dass sich das ärztliche und pflegerische Ethos anpasst, dass etwa das Patientenwohl nicht mehr fraglos an erster Stelle steht, sondern als gleichrangig mit ökonomischen Erwägungen wahrgenommen wird.

Solche Aspekte in Begleitforschung einzubeziehen, kann interessante Ergebnisse zutage fördern. So berichtet eine Studie im Auftrag der FMH, dass ein Drittel der teilnehmenden akutsomatischen Ärztinnen und Ärzte beobachtet hat, dass «versucht wird, chronisch kranke und multimorbide Patienten in anderen Einrichtungen unterzubringen» [4]. In der IDoC-Studie, deren erste Ergebnisse sich im Publikationsprozess befinden, hat sich gezeigt, dass die ärztlichen Studienteilnehmer keine Effizienzsteigerung seit der Einführung von SwissDRG, zugleich aber eine nachlassende Patientenorientierung und durchaus verfrühte Entlassungen aus ökonomischen Gründen sowie andere Formen von Unter- und Überversorgung wahrnehmen [5].

Vor kurzem hat ein IDoC-Symposium stattgefunden, auf dem sich ein Spektrum an Akteuren versam- melt hat, um die vorläufigen Ergebnisse des Projekts zu diskutieren. Dabei kristallisierten sich folgende Einsichten heraus:

- Für die Erfassung und Beurteilung mancher DRG-Effekte ist ein längerfristiger Beobachtungszeitraum notwendig.

- Der Einbezug der Wahrnehmung von medizinischem Fachpersonal ist zwar wichtig, doch sollten darüber hinaus auch die Erfahrungen von Patientinnen und Patienten einfliessen. Die systematische Erhebung von Patientenzufriedenheit ist dafür eine wichtige Voraussetzung.

- Was mit der Einführung der DRGs passiert, wird entscheidend durch die Weise geprägt, wie diese im betreffenden Spital umgesetzt werden. Damit rücken Fragen einer angemessenen «Kultur» des Umgangs mit einem verstärkten Fokus auf ökonomische Faktoren und mögliche resultierende Interessenkonflikte in den Blick.

- Schliesslich wurde deutlich, dass die Debatte über DRGs hinaus auf Unter-, Über- und Fehlversorgung verweist, die - wenn auch in unterschiedlichen Spielarten - unter allen möglichen Abrechnungssystemen auftreten kann.

Die Chance der DRGs besteht darin, unser Bewusstsein für die Konsequenzen von Anreizsystemen zu schärfen und Anreize immer besser so auszutarieren, dass Effizienzgewinne ohne Einbussen an Qualität und Fairness der Gesundheitsversorgung erfolgen. Dabei ist klar: DRGs, wie andere Formen der Vergütung auch, sind als lernende Syteme zu verstehen, die nicht nur möglichst reichhaltiger Daten als Rückmeldung für die weitere Optimierung bedürfen, sondern sich auch einer fortlaufenden Evaluation stellen müssen.

Dabei geht es nicht um die Frage, ob DRGs «gut» oder «schlecht» sind, sondern darum, ob sie das Ziel einer guten Gesundheitsversorgung für alle zu vernüftigen, nachhaltig leistbaren Kosten wirksam befördern und damit die hohe Performanz des schweizerischen Gesundheitssystems auch in der Zukunft gewährleisten können. Hierzu trägt IDoC durch die Bereitstellung methodischer Instrumente und erster empirischer Befunde bei.

\section{Referenzen}

1 Botschaft betreffend die Änderung des Bundesgesetzes über die Krankenversicherung (Spitalfinanzierung) vom 15. September 2004. www.admin.ch/opc/de/ federal-gazette/2004/5551.pdf, letzter Zugriff am 19.1.2014.

2 Engler C. Guter Start mit neuen Fallpauschalen. Kurzinformationen von H+. 2012;1:3.

3 Schoch C. Keine Zunahme von blutigen Spitalaustritten. Neue Zürcher Zeitung, 8. Januar 2014.

4 Meyer B, Gider L, Longchamp C. Hohe Berufsidentifikation trotz steigender Bürokratie. Schweiz Ärztezeitung. 2014;95(1/2):7-8.

5 Friedli D. Fallpauschalen mit blutigen Folgen. NZZ am Sonntag, 24. November 2013. 